\title{
Individualized Web-Based Attention Training With Evidence-Based Counseling to Address HIV Treatment Adherence and Psychological Distress: Exploratory Cohort Study
}

Eric Houston $^{1,2}$, PhD; Javad Salehi Fadardi ${ }^{1}, \mathrm{PhD}$; Nina T Harawa ${ }^{2,3}, \mathrm{PhD}$; Chris Argueta ${ }^{4}$, BA; Sukrit Mukherjee ${ }^{3}$, $\mathrm{PhD}$

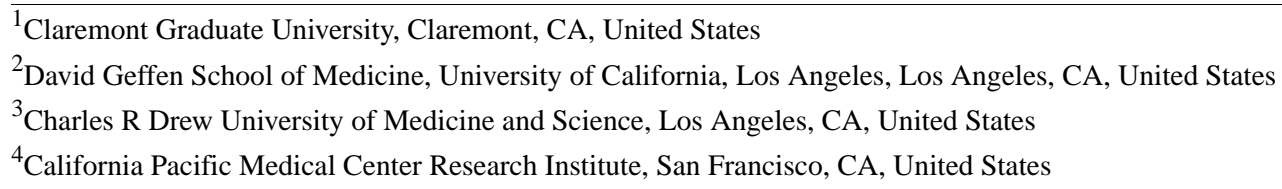

Corresponding Author:

Eric Houston, $\mathrm{PhD}$

Claremont Graduate University

675 West Foothill Boulevard

Claremont, CA, 91711

United States

Phone: 19096218000

Email: eric.houston@cgu.edu

\section{Abstract}

Background: The prevalence of mood, trauma, and stressor-related disorders is disproportionately higher among people living with HIV than among individuals without the virus. Poor adherence to HIV treatment and heightened psychological distress have been linked to symptoms associated with these disorders.

Objective: The objective of this exploratory pilot study was to develop and implement an intervention that combined individualized web-based attention training with evidence-based counseling to promote HIV treatment adherence and reduce psychological distress among people living with HIV. The study targeted African American and Latino young men who have sex with men, two population groups in the US that continue to experience disparities in HIV treatment outcomes.

Methods: Study participants with elevated symptoms of depression and suboptimal adherence to antiretroviral therapy were recruited primarily through referrals from Los Angeles health and social service providers as well as postings on social media. Participants enrolled in the 4-week intervention received weekly counseling for adherence and daily access to web-based attention training via their personal mobile devices or computers.

Results: Of the 14 participants who began the intervention, $12(86 \%)$ completed all sessions and study procedures. Using a pretest-posttest design, findings indicate significant improvements in adherence, depressive symptoms, and attention processing. Overall, the proportion of participants reporting low adherence to antiretroviral therapy declined from $42 \%$ at baseline to $25 \%$ at intervention completion $(P=.02$, phi=0.68). Mean depressive symptoms measured by the 9 item Patient Health Questionnaire (PHQ-9) showed a substantial reduction of $36 \%(P=.002$, Cohen $d=1.2)$. In addition, participants' attentional processing speeds for all types of stimuli pairings presented during attention training improved significantly $(P=.01$ and $P=.02)$ and were accompanied by large effect sizes ranging from 0.78 to 1.0 .

Conclusions: Our findings support the feasibility of web-based attention training combined with counseling to improve antiretroviral therapy adherence among patients with psychological distress. Future research should include a larger sample, a control group, and longer-term follow-up.

(JMIR Ment Health 2021;8(1):e18328) doi: 10.2196/18328

\section{KEYWORDS}

depression; trauma; HIV; attention training; implicit cognition 


\section{Introduction}

People living with HIV are disproportionately affected by depression and posttraumatic stress disorder (PTSD) [1,2]. Compared to the general population, studies have estimated prevalence rates among people living with HIV at two to three times higher for depression and up to nine times higher for PTSD [3,4]. While both disorders adversely affect adherence to antiretroviral therapy, symptoms of depression and PTSD, even at subclinical levels, weaken an individual's ability to effectively self-regulate the attention and cognitive processes required for consistent goal-directed behavior, such as following a long-term treatment regimen [5-7].

Research indicates that poor antiretroviral therapy adherence and engagement with HIV medical care are connected to difficulties that HIV patients face in controlling negative thoughts, memories, and impulses to effectively manage their attentional focus [8-12]. To meet the challenges associated with attaining their treatment goals, patients must learn to effectively employ cognitive self-regulatory skills, including attention control and the ability to flexibly shift attentional focus from emotionally negative health-compromising thoughts toward those that are positive or neutral and associated with favorable health outcomes $[13,14]$.

Little research has focused on the development of interventions designed specifically to build the cognitive self-regulatory skills needed for optimal antiretroviral therapy adherence. There is, however, a growing body of empirical and theoretical evidence that demonstrates the efficacy of attention training approaches in developing these skills, thereby promoting consistent goal-directed behavior related to a wide variety of health concerns, including smoking, problem drinking, substance abuse, eating disorders, being overweight, and obesity [15-17]. Similarly, attention training procedures have demonstrated efficacy in addressing several mental health problems for both adults and adolescents, including anxiety disorders and major depression [18-21]. Such approaches provide structured training designed to strengthen an individual's ability to shift his or her attentional focus away from stimuli that provoke thoughts and memories associated with treatment avoidance and psychological distress and toward stimuli that promote treatment engagement and emotional well-being. In addition, the indirect nature of attention training approaches makes them appropriate for addressing the implicit or nonconscious thoughts, beliefs, and memories that are related to depression, anxiety, and trauma $[22,23]$.

Despite promising research indicating the value of attention training in addressing both psychological and physical health outcomes, there is a paucity of research focused on the application of attention training among HIV patients with comorbid psychiatric symptoms [24]. Attention training is a clinically relevant approach given that negative attention biases are closely linked to depressed mood and a tendency for threat vigilance in connection with anxiety and trauma. The training of attention away from salient negative stimuli and toward neutral or positive stimuli could be used to improve treatment adherence, increase engagement with HIV medical care, and promote mental health functioning. To enhance treatment outcomes and the durability of attention training effects, individualized stimuli could be presented during each session, thereby addressing the salient thoughts, beliefs, memories, and images that trigger specific types of behavior or emotional responses for a given patient. Research indicates that individualized stimuli trigger stronger attention biases among study participants than general stimuli $[25,26]$.

We conducted an exploratory pilot study to develop and implement an intervention consisting of a 4-week, web-based attention training program combined with evidence-based counseling to improve antiretroviral therapy adherence and reduce psychological distress among HIV patients. Individualized, web-based attention training, in combination with evidence-based counseling, has the potential to serve as an accessible approach that could be widely disseminated to reach individuals at risk for poor treatment outcomes due to suboptimal antiretroviral therapy adherence or psychological distress. As a first step in exploring this potential role, the study sought to develop and determine whether such an intervention approach could be efficiently deployed and conveniently delivered to patient populations disproportionately affected by HIV. African American and Latino young men who have sex with men represent two vulnerable patient populations in the US that suffer from persistent elevated viremia, disproportionately high HIV transmission rates, and low levels of engagement in the HIV care continuum [27]. Given the need for effective cognitive self-regulatory skills in maintaining consistent adherence and engagement with care amid elevated psychological distress and multiple psychosocial stressors (eg, HIV stigma, experiences of trauma, childhood abuse), the study provided an opportunity to evaluate the viability of intervention featuring web-based individualized attention training to reach these vulnerable patient populations.

\section{Methods}

\section{Participants}

The sample consisted of participants recruited for Project STEP (Steps Toward Embodying Positivity), an intervention designed to address HIV treatment adherence and depressive symptoms among African-American and Latino young men living with HIV in the Los Angeles metropolitan area. Participants in the intervention, which combined individualized web-based attention training with evidence-based counseling, were recruited primarily through referrals from health and social service providers and through postings on social media used by the target population (eg, Adam4Adam, Craigslist, Grindr).

Individuals who expressed interest in joining the study were screened in person or by phone to determine if they met the following 4 eligibility criteria: (1) African American or Latino male living with HIV; (2) 18- 29 years old, inclusive; (3) self-identified as gay, bisexual, or same-gender loving; and (4) depressive symptoms at mild or higher levels of severity based on self-report measures or suboptimal antiretroviral therapy adherence, two psychological and behavioral risk factors for poor HIV treatment outcomes that the intervention was designed to address. 


\section{Procedure}

Upon meeting the inclusion criteria, participants were administered informed consent and then enrolled in the study. Assessments and interviews with participants were conducted by a study team member during face-to-face meetings. After completing procedures to elicit and assess their individualized stimuli (ie, brief thoughts related to treatment and mood changes), participants were scheduled for a second study meeting in which they were given an attention training tutorial and assessed for baseline reaction time performance. Upon finishing 4 weeks of attention training, participants were scheduled for a posttraining assessment within a week of their final training session. Participants received $\$ 50$ each for completion of the baseline and final assessments and $\$ 5$ for each weekly meeting attended. All procedures for recruitment, data collection, and confidentiality were reviewed and approved by the Institutional Review Board of Charles R. Drew University of Medicine and Science.

\section{Measures}

\section{Overview}

We collected study data through use of a self-administered computerized survey that participants completed during baseline and posttraining study visits. To gauge the preliminary impact of the intervention, the computerized survey included measures of adherence and depressive symptoms. To describe our sample, we administered questionnaires tapping demographic and health-related characteristics, anxiety and trauma symptoms, and psychosocial stressors (eg, HIV stigma and childhood sexual abuse). On average, participants completed survey items within 30 minutes.

\section{Demographics}

Participants provided basic sociodemographic data by completing a 22-item questionnaire that requested information related to age, ethnicity, education, employment, income, HIV serostatus, healthcare usage, and other personal characteristics.

\section{Adherence}

Self-reported adherence was assessed using a modified version of the visual analogue scale (VAS) [28,29]. In the scale used in this study, participants were presented with a horizontal number line divided into 4 segments to represent the percentage of HIV medication doses missed during the 4 days prior to the assessment (ie, 0\%-25\%; 25\%-50\%; 50\%-75\%; and 75\%-100\%). Using the number line, participants were instructed to indicate their adherence within 1 of the 4 categories. The final category encompassed moderate and high levels of adherence. Recent research suggests that newer formulations of antiretroviral therapy may allow some patients to achieve virologic suppression and immunological benefits with relatively moderate adherence levels [30-34]. The modified version of the scale was used to enhance its administration and reduce response bias.

\section{Depressive Symptoms}

Depressive symptoms during the 2 weeks prior to assessment were measured using the Patient Health Questionnaire-9 (PHQ-9) [35]. The PHQ-9 is a well-validated and widely-used brief instrument for assessing and monitoring depression severity. Depression scores derived from the PHQ-9 correspond to minimal $(\leq 4)$, mild (5-9), moderate (10-14), moderately severe (15-19), or severe ( $\geq 20)$. Based on systematic reviews and a meta-analysis of the PHQ-9, a cutoff score of 10 or greater has been described as indicative of meeting diagnostic criteria for depression $[35,36]$. The instrument had a Cronbach alpha of .89 .

\section{Trauma Symptoms}

The Posttraumatic Stress Checklist-Civilian Version [37] was used to measure trauma symptoms. The checklist is a 17 -item self-report measure of PTSD symptoms. Participants were asked to respond to each item using a 5-point Likert scale response format. Scores on the instrument range from 17 to 85 , with higher scores indicating greater symptom severity. A score of 30 has been recommended as the minimum threshold for the further evaluation of PTSD symptoms among individuals in a civilian population [38].

\section{Anxiety}

The Modified Mini Screen [39] was administered to gauge anxiety symptoms. It is a 22-item scale designed to identify individuals who may have psychiatric symptoms at levels that warrant further evaluation. We used 9 items from the scale to assess anxiety symptoms among participants, with scores of 6 or greater indicating elevated levels of anxiety.

\section{Childhood Sexual Abuse}

Two items from the Child Sexual Abuse Index [40] were used to identify participants who had been subjected to sexual abuse during childhood. Specifically, participants were asked to indicate whether before the age of 18 they experienced (1) unwanted sexual events and/or (2) sexual abuse or molestation. The Child Sexual Abuse Index also includes additional items in which participants indicated the type of sexual abuse, whether it involved violence or physical force, their age when the abuse occurred, and their relationship to the perpetrator(s).

\section{HIV Stigma}

To assess the presence of HIV stigma, we used the AIDS-Related Stigma Scale [41]. Participants were asked to respond (yes/no) to 6 dichotomous items pertaining to internalized negative beliefs and perceptions about people living with HIV.

\section{Attention Training: Project STEP}

The goal of attention training through Project STEP was twofold. First, it was designed to increase treatment adherence by teaching participants how to maintain their focus on thoughts that were approach-oriented with regard to treatment and to direct their focus away from thoughts that were avoidance-oriented. Second, to reduce depressive symptoms, attention training also sought to increase a participant's skill in quickly diverting attention away from those thoughts perceived as having an emotionally-negative valence and directing attention toward thoughts that were perceived as neutral or having an emotionally-positive valence. Attention training, delivered via a web-based app, used the participant's own 
thoughts identified during an individualized assessment procedure.

During the assessment procedure, we elicited a wide range of personal thoughts and perceptions about treatment from participants through individual interviews. Thoughts related to positive and negative changes in the participants' emotional states were also elicited. Individual interviews were followed by administration of a computerized program in which participants were asked to quickly rate the similarity of paired combinations of their treatment-related thoughts as they appeared in random order on the computer screen. The computerized rating procedure is consistent with other research designed to identify implicit cognitive processes [42-44]. Ratings were subjected to multidimensional scaling analysis to generate 2D mappings that depicted how a participant's treatment-related thoughts, memories, and mental associations were associated with either treatment adherence or treatment avoidance. Multidimensional scaling analysis has been used and evaluated as an approach for the assessment of implicit cognitive processes $[8,43,45]$. Both implicit and explicit cognitive processes were captured through the assessment procedure.

\section{Modified Dot-Probe Task}

Attention training was delivered through a modified version of the dot-probe task, a spatially oriented computerized procedure employed to retrain attentional focus. Using a web-based version of the task developed for the present study, participants accessed the dot-probe task via their computer or mobile device and completed sessions at home. At the start of a training session trial, participants were asked to view the screen of their device and watch a fixation cross that was situated in the center of the screen. After $1000 \mathrm{~ms}$, two stimuli consisting of contrasting thoughts that were elicited during individualized assessments replaced the cross and appeared simultaneously on opposite sides of the screen for approximately $2500 \mathrm{~ms}$. Then, a dot-probe appeared on the screen in the location of one of the previous stimuli. At this time, participants were required to indicate the location of the dot-probe as quickly as possible by clicking on their cursor or touching the screen of their device. The probe always appeared in the location of the stimuli that were treatment approach-oriented and conveyed a neutral or positive emotional tone, thereby training participants to respond to these types of stimuli rather than to negative and treatment avoidance-oriented stimuli.

Each individualized training session lasted approximately 15 minutes and was presented in 4 blocks. A single training block was composed of 50 trials, with a trial consisting of each sequence from the appearance of the fixation cross to the onset of the dot-probe. Completion of a training session required that the participant finish all 4 blocks. Participants, who received a tutorial practice session on the use of the attention training program prior to beginning the intervention, were provided information on the rationale behind attention training, an explanation of attention training procedures, and explicit instructions in which both speed and accuracy were emphasized. To ensure they understood how to use the computerized program, participants were required to have an accuracy rate of
$80 \%$ during the tutorial practice session before proceeding to actual intervention training. During the intervention, trial-by-trial feedback in the form of an audible signal alert was provided during attention training sessions to aid participants in reorienting their attentional focus. At the end of each block of training, participants were presented with a screen that showed their reaction time and accuracy rate for that specific training session. Participants were asked to complete at least three individualized training sessions at home on a daily basis for 4 consecutive weeks. With repeated trials, participants were expected to implicitly learn how to redirect or retrain their attentional focus toward neutral, positive, and approach-oriented stimuli and away from treatment avoidance-oriented stimuli associated with negative emotional states and poor health behaviors. To evaluate changes in the amount of time a participant required to shift their attentional focus from avoidance-oriented or negative thoughts toward those that were approach-oriented or positive/neutral (ie, attentional processing speed), we used reaction time measures collected during baseline and posttraining administrations of the modified dot-probe task.

\section{Weekly Counseling}

In addition to attention training, participants received weekly counseling related to HIV treatment adherence and cognitive self-regulation. Two intervention counselors, who matched the age, gender, and ethnic characteristics of the sample, were trained and supervised by the principal investigator, a licensed clinical psychologist. The first of the 4 counseling sessions focused on psychoeducational content, such as the role of thoughts in health behavior and affect. Participants were given information on techniques to identify and monitor their thoughts and were encouraged to discuss how attention training could be used to effectively manage their thought processes. During the subsequent 3 meetings, participants were presented with selected modules adapted from the Treatment Advocacy Program [46-48], an evidence-based individual level counseling intervention for people living with HIV. Modules were delivered by counselors in the form of PowerPoint slides via a laptop computer or iPad. Treatment Advocacy Program modules selected for this study provided participants with behavioral strategies and information pertaining to antiretroviral therapy adherence, mood management, and alcohol and substance use. Participants were also given information on local resources and provided with referrals when needed.

\section{Data Analytic Strategy}

Data analysis was performed using IBM SPSS 22.0. Due to the exploratory nature of the study and the corresponding small sample size, data analysis focused primarily on descriptive statistics. The full sample consisted of individuals who were enrolled into the study and completed baseline questionnaires. We examined data from the full sample $(\mathrm{N}=20)$ to characterize participants who met eligibility criteria and completed baseline measures. Most analyses presented in this report, however, are based on data from participants who completed the attention training intervention and final assessments $(n=12)$. To compare participant characteristics based on study completion status, we used the Chi-square statistic for categorical variables and independent samples $t$-test for continuous variables. The 
Chi-square statistic was also used to examine changes in adherence among participants from pre- to posttraining assessments.

We used $t$-tests to examine changes in mean numbers of depressive symptoms, reaction times, and accuracy scores. Reaction time analyses included reaction times only from correct responses. To reduce the influence of outliers, we eliminated reaction times that were 1.5 standard deviations above or below a participant's mean response time. This approach is consistent with other published research [49]. Effect sizes were calculated using the phi statistic for categorical variables ( small effect $=0.1$; medium effect $=0.3$; large effect $=0.5$ ) and Cohen $d$ for comparisons of means ( mall effect $=0.2$; medium effect $=0.5$; large effect=0.8) [50].The alpha level for all statistical tests was set at .05 .

\section{Results}

\section{Sample Description}

African Americans comprised the majority of the full sample $(12 / 20,60 \%)$ and Latinos represented $40 \%$ (8/20). Mean participant age was 27 years (SD 1.7). Study participants identified as gay $(15 / 20,75 \%)$ or bisexual $(5 / 20,25 \%)$. Sixty-five percent of participants (13/20) indicated that they had completed high school, a high school equivalency credential, or some college, and $20 \%$ (4/20) reported graduating from college. Fifty-five percent of the full sample (11/20) had annual incomes below $\$ 20,000$.

Adherence in the full sample was considerably below optimal levels, with the majority of participants $(12 / 20,60 \%)$ reporting adherence rates less than or equal to $75 \%$. Participants experienced high levels of psychological distress. The mean depressive symptom score was 11.95 (SD 6.6), which is in the moderate range based on the PHQ-9 and is above the threshold widely used to suggest further evaluation for major depression. Fifty-five percent of participants in the full sample (11/20) reported elevated symptoms of anxiety. With regard to trauma symptoms, 40 percent $(8 / 20)$ had symptoms at or above recommended screening level cutoffs for PTSD. Forty-five percent (9/20) reported unwanted sexual events, sexual abuse, or molestation before the age of 18 . Eighty percent $(16 / 20)$ reported experiencing internalized HIV stigma.

Of the 20 participants enrolled, 6 were excluded as they failed to attend a required study meeting for orientation to attention training procedures. Based on the remaining 14 participants who began attention training, the study completion rate was $86 \%$ (2 participants dropped out for unknown reasons before completing the protocol). There were no statistically significant differences between enrolled participants who completed the intervention and those who were excluded or dropped out with regard to demographics, adherence, depressive symptoms, or other assessed variables.

\section{Attention Training}

Participants were encouraged to complete at least three attention training sessions on a daily basis, for a total of 84 sessions during the intervention. The median number of training sessions among intervention completers was 48 . All 12 study participants who completed the intervention attended each of the 4 weekly meetings with a study counselor.

Table 1 shows changes in attentional processing speed among study participants. Attentional processing was based on mean reaction times scores grouped by assessment period (baseline vs posttraining) and by stimuli pairing type (ie, positive-neutral, negative-neutral, positive-negative, and neutral-neutral). Participant reaction times to correctly identify the location of the dot-probe in each of the 4 stimuli pairings significantly declined from baseline to posttraining assessments, with large Cohen $d$ effect sizes ranging 0.78 to 1.0. Participants experienced the greatest mean reduction in reaction times for the positive-negative stimulus pairings $(369 \mathrm{~ms})$, followed by positive-neutral pairings (353 $\mathrm{ms})$.

Table 1. Mean dot-probe reaction time to stimuli presented at baseline and posttraining assessments $(\mathrm{n}=12)$.

\begin{tabular}{lllll}
\hline Stimuli pairing & Baseline mean reaction time, ms (SD) & Posttraining mean reaction time, ms (SD) & Test statistic & $P$ value \\
\hline Positive-neutral & $2553(693)$ & $2200(375)$ & -3.25 & .01 \\
Negative-neutral & $2579(770)$ & $2255(414)$ & -2.71 & .02 \\
Positive-negative & $2561(677)$ & $2192(368)$ & -3.48 & .01 \\
Neutral-neutral & $2483(683)$ & $2175(331)$ & -3.23 & .01 \\
\hline
\end{tabular}

Accuracy scores for each of the 4 types of stimuli pairings during the baseline and posttraining assessments were calculated to provide a measure of the rate at which participants correctly responded when prompted to indicate the location of the dot-probe. The only statistically significant change in accuracy scores from baseline to posttraining assessments, however, occurred when participants responded to positive-negative stimuli pairings ( $94 \%$ vs $98 \%$, respectively; $t_{10}=4.05, P=.002$ ).

\section{Treatment Adherence}

We examined the relationship between attention training and antiretroviral therapy adherence before and after the intervention by examining participant adherence rates based on a threshold of $75 \%$. We categorized participants with adherence at or below this threshold as having "low" adherence and those with adherence above the threshold as having "moderate/high" adherence. In the current study, participants with low adherence reported rates between $50 \%$ to $75 \%$. At baseline, $42 \%(5 / 12)$ of participants who completed the intervention had low adherence versus 58\% (7/12) with moderate/high adherence. During the posttraining assessment, however, these percentages had shifted significantly with $75 \%$ (9/12) reporting adherence in the moderate/high range (Table 2). This change in adherence represented a large effect size (phi coefficient=.68). 
Table 2. Changes in antiretroviral therapy adherence and number of depressive symptoms among intervention participants ( $\mathrm{n}=12$ ).

\begin{tabular}{|c|c|c|c|c|}
\hline Characteristic & Baseline & Posttraining & Test statistic & $P$ value \\
\hline Adherence rate, $\mathrm{n}(\%)$ & & & 5.6 & .02 \\
\hline$\leq 75 \%$ & $5(42)$ & $3(25)$ & $\mathrm{N} / \mathrm{A}^{\mathrm{a}}$ & N/A \\
\hline$>75 \%$ & $7(58)$ & $9(75)$ & $\mathrm{N} / \mathrm{A}$ & N/A \\
\hline Depressive symptoms, mean (SD) & $13.4(6.8)$ & $8.6(7.5)$ & 3.71 & .002 \\
\hline \multicolumn{5}{|l|}{ Symptom severity, n (\%) } \\
\hline Minimal (0-4) & $1(8)$ & $3(25)$ & N/A & N/A \\
\hline Mild (5-9) & $3(25)$ & $6(50)$ & N/A & N/A \\
\hline Moderate (10-14) & $2(17)$ & $0(0)$ & N/A & N/A \\
\hline Moderately-severe (15-19) & $4(33)$ & $2(17)$ & N/A & N/A \\
\hline
\end{tabular}

${ }^{\mathrm{a}}$ N/A: not applicable.

\section{Depressive Symptoms}

Mean depressive symptoms among participants who completed the intervention declined significantly by $36 \%$ based on the PHQ-9, from 13.4 (SD 6.8) at baseline to 8.6 (SD 7.5) posttraining, $\left(t_{11}=4.16, P=.002\right.$; Cohen $d=1.2$, indicating a large effect size). The mean baseline score, which was in the moderate range with regard to depressive symptom severity, exceeded the cutoff of 10 widely used to suggest further diagnostic evaluation for major depression. The mean posttraining score, which fell below this cutoff, was clinically significant in that it represented an overall downward shift in symptom severity from the moderate to the mild range. As Table 2 shows, compared to baseline levels, there was a marked drop in the percentages of participants who experienced depressive symptoms in the moderate, moderate-severe, and severe ranges upon completing the study.

\section{Discussion}

\section{Principal Findings}

In this exploratory pilot study, we aimed to develop and implement an intervention consisting of individualized, web-based attention training combined with evidence-based counseling to promote adherence to antiretroviral therapy and reduce depressive symptoms among HIV patients experiencing elevated levels of psychological distress. Findings indicate that web-based attention training in addition to counseling can be efficiently deployed and conveniently delivered to a vulnerable HIV patient population with suboptimal antiretroviral therapy adherence and disproportionately high rates of depressive and PTSD symptoms. The intervention implemented in this study had a high completion rate $(12 / 14,86 \%)$, indicating strong viability as a clinical approach. Participants were able to access an individualized attention training program through their own mobile devices or computers, completing a median of 48 sessions during the 4-week attention training program, or the equivalent of approximately two sessions per day. Attention training combined with evidence-based counseling yielded considerable therapeutic benefits to intervention participants. We found statistically significant improvements among participants in antiretroviral therapy adherence from pre- to posttraining. In addition, we found both statistically and clinically significant reductions in depressive symptoms. Findings also showed notable improvements in attentional processing speed based on reaction time measures. Research suggests that improvements in processing speed play an important role in promoting everyday functioning and quality of life [51,52].

While participants in this study were assessed at only two time intervals, the study effectively employed strategies that could be used to maximize the benefits of attention training and strengthen the long-term durability of intervention outcomes. Our intervention used specific strategies (eg, performance feedback) to enhance the learning experience of study participants, drawing from recent research involving attention training [53-62]. Based on goal setting theory [63], these strategies included providing participants with explicit instructions, a clear statement of the training goal, and trial-by-trial feedback on performance (eg, reaction time changes; response accuracy rate). This study illustrates how individualized, web-based attention training for HIV patients with psychological distress could be employed in combination with psychotherapy.

Participants represented individuals who could most benefit from attention training due to the cognitive burden posed by multiple psychosocial stressors (eg, clinically-significant depressive symptoms, experiences of trauma and abuse, and internalized HIV stigma). Future studies, however, should be based on larger samples that include women and individuals representing a wider range of ages, geographic locations, and behavioral risk groups. Although studies support the validity of self-report measures of adherence [64], findings in this investigation could be bolstered by future research that incorporates biomedical measures of adherence. In addition, future studies should be designed to examine measures of cognitive self-regulation related to attention control, cognitive flexibility, and attention bias. Such measures would be derived based on administration of a standard dot-probe task where all stimuli are targeted with equal probability. This preliminary pilot study did not administer the standard dot-probe task. 
Finally, to better understand the role of attention training in HIV patient outcomes, research should be conducted that tracks participants over longer time intervals with a design that incorporates other approaches and a control group.

Attention training has shown much promise as an approach to improve outcomes associated with a range of health behaviors and psychological disorders [15-21,24,65]. This exploratory study contributes to the literature on attention training by showing its clinical applications in addressing the impact of depressive and trauma symptoms on HIV treatment adherence.
We were able to provide evidence of the ability of individualized, web-based attention training to yield favorable improvements in adherence and psychological distress in two vulnerable populations of HIV patients. Our findings provide support for additional exploration of this promising application.

\section{Data Availability Statement}

The raw data supporting the conclusions of this manuscript will be made available by the authors, without undue reservation, to any qualified researcher.

\section{Acknowledgments}

The authors would like to thank Kieran Breda-Hill, Wendell Glenn, Jahmil Lacey, Luis Mancilla, Kelvin Sterling Scott, Alan Stacy, and the Project STEP advisory board (Alonso Bautista, Juan Carmen, Antonio Castaneda, Thomas Davis, Abraham Estrada, Donta Morrison, and Maribel Prosas) for their assistance and support in completing this report.

The first author is supported by a grant from the National Institute on Minority Health and Health Disparities (NIMHD; S21 MD000103; David M Carlisle, MD, Principal Investigator). This research was supported by Grant 5U54MD007598 from NIMHD (Jaydutt V Vadgama, PhD, Principal Investigator).

\section{Authors' Contributions}

EH conceived and designed the study, performed data analysis, and wrote the manuscript. JSF and NTH contributed to the writing of the manuscript. CA performed data collection, organized the database, and performed data entry. SM developed attention training software and contributed to data collection activities. All authors agreed on the final manuscript.

\section{Conflicts of Interest}

None declared.

\section{References}

1. Bing EG, Burnam MA, Longshore D, Fleishman JA, Sherbourne CD, London AS, et al. Psychiatric disorders and drug use among human immunodeficiency virus-infected adults in the United States. Arch Gen Psychiatry 2001 Aug 01;58(8):721-728. [doi: 10.1001/archpsyc.58.8.721] [Medline: 11483137]

2. Israelski DM, Prentiss DE, Lubega S, Balmas G, Garcia P, Muhammad M, et al. Psychiatric co-morbidity in vulnerable populations receiving primary care for HIV/AIDS. AIDS Care 2007 Feb 11;19(2):220-225. [doi: 10.1080/09540120600774230] [Medline: 17364402]

3. Applebaum AJ, Bedoya AC, Hendriksen ES, Wilkinson JL, Safren SA, O'Cleirigh C. Future directions for interventions targeting PTSD in HIV-infected adults. J Assoc Nurses AIDS Care 2015;26(2):127-138 [FREE Full text] [doi: 10.1016/j.jana.2014.11.001] [Medline: 25665885]

4. Chander G, Himelhoch S, Moore RD. Substance abuse and psychiatric disorders in HIV-positive patients: epidemiology and impact on antiretroviral therapy. Drugs 2006;66(6):769-789. [doi: 10.2165/00003495-200666060-00004] [Medline: $\underline{16706551]}$

5. Blashill AJ, Perry N, Safren SA. Mental health: a focus on stress, coping, and mental illness as it relates to treatment retention, adherence, and other health outcomes. Curr HIV/AIDS Rep 2011 Dec 6;8(4):215-222 [FREE Full text] [doi: 10.1007/s11904-011-0089-1] [Medline: 21822626]

6. Boarts JM, Sledjeski EM, Bogart LM, Delahanty DL. The differential impact of PTSD and depression on HIV disease markers and adherence to HAART in people living with HIV. AIDS Behav 2006 May 16;10(3):253-261. [doi: 10.1007/s10461-006-9069-7] [Medline: 16482405]

7. Vranceanu AM, Safren SA, Lu M, Coady WM, Skolnik PR, Rogers WH, et al. The relationship of post-traumatic stress disorder and depression to antiretroviral medication adherence in persons with HIV. AIDS Patient Care STDS 2008 Apr;22(4):313-321. [doi: 10.1089/apc.2007.0069] [Medline: 18338960]

8. Houston E, Lyons T, Wolfe B, Rolfsen N, Williams M, Rucker M, et al. Assessing Implicit Cognition Among Patients Lost to Follow-up for HIV Care: A Preliminary Study. Open AIDS J 2016 May 09;10(1):83-92 [FREE Full text] [doi: 10.2174/1874613601610010083] [Medline: 27347274]

9. McIntosh RC, Tartar JL, Widmayer S, Rosselli M. Negative attention bias and processing deficits during the cognitive reappraisal of unpleasant emotions in HIV+ women. J Neuropsychiatry Clin Neurosci 2015 Jan;27(1):e32-e39. [doi: 10.1176/appi.neuropsych.13090222] [Medline: 25541865]

10. Novara C, Casari S, Compostella S, Dorz S, Sanavio E, Sica C. Coping and cognitive processing style in HIV-positive subjects. Psychother Psychosom 2000 Nov 3;69(6):316-321. [doi: 10.1159/000012414] [Medline: 11070444] 
11. St Quinton T, Brunton JA. Implicit Processes, Self-Regulation, and Interventions for Behavior Change. Front Psychol 2017 Mar 08;8:346 [FREE Full text] [doi: 10.3389/fpsyg.2017.00346] [Medline: 28337164]

12. Webb TL, Chang BPI, Benn Y. 'The Ostrich Problem': Motivated Avoidance or Rejection of Information About Goal Progress. Social and Personality Psychology Compass 2013 Nov 04;7(11):794-807. [doi: 10.1111/spc3.12071]

13. Hall PA, Marteau TM. Executive function in the context of chronic disease prevention: theory, research and practice. Prev Med 2014 Nov;68:44-50. [doi: 10.1016/j.ypmed.2014.07.008] [Medline: 25042899]

14. Satpute A, Ochsner K, Badre K. The neuroscience of goal-directed behavior. In: Aarts H, Elliot AJ, editors. Goal-directed behavior. New York: Psychology Press; 2012:49-83.

15. Cox WM, Fadardi JS, Intriligator JM, Klinger E. Attentional bias modification for addictive behaviors: clinical implications. CNS Spectr 2014 Jun;19(3):215-224. [doi: 10.1017/S1092852914000091] [Medline: 24642267]

16. Kakoschke N, Kemps E, Tiggemann M. Attentional bias modification encourages healthy eating. Eat Behav 2014 Jan;15(1):120-124. [doi: 10.1016/j.eatbeh.2013.11.001] [Medline: 24411764]

17. Lopes FM, Pires AV, Bizarro L. Attentional bias modification in smokers trying to quit: a longitudinal study about the effects of number of sessions. J Subst Abuse Treat 2014 Jul;47(1):50-57. [doi: 10.1016/j.jsat.2014.03.002] [Medline: 24666812]

18. Beevers CG, Clasen PC, Enock PM, Schnyer DM. Attention bias modification for major depressive disorder: Effects on attention bias, resting state connectivity, and symptom change. J Abnorm Psychol 2015 Aug;124(3):463-475 [FREE Full text] [doi: 10.1037/abn0000049] [Medline: 25894440]

19. Kuckertz JM, Amir N, Boffa JW, Warren CK, Rindt SE, Norman S, et al. The effectiveness of an attention bias modification program as an adjunctive treatment for Post-Traumatic Stress Disorder. Behav Res Ther 2014 Dec;63:25-35 [FREE Full text] [doi: 10.1016/j.brat.2014.09.002] [Medline: 25277496]

20. Yang W, Ding Z, Dai T, Peng F, Zhang JX. Attention Bias Modification training in individuals with depressive symptoms: A randomized controlled trial. J Behav Ther Exp Psychiatry 2015 Dec;49(Pt A):101-111 [FREE Full text] [doi: 10.1016/j.jbtep.2014.08.005] [Medline: 25245928]

21. Zhang M, Ying J, Song G, Fung DSS, Smith H. Web-Based Cognitive Bias Modification Interventions for Psychiatric Disorders: Scoping Review. JMIR Ment Health 2019 Oct 24;6(10):e11841 [FREE Full text] [doi: 10.2196/11841] [Medline: $\underline{31651410]}$

22. Bar-Haim Y. Research Review: attention bias modification (ABM): a novel treatment for anxiety disorders. J Child Psychol Psychiatry. 51 2010:859. [doi: 10.1111/j.1469-7610.2010.02251.x]

23. Joormann J, Quinn ME. Cognitive processes and emotion regulation in depression. Depress Anxiety 2014 Apr 25;31(4):308-315. [doi: 10.1002/da.22264] [Medline: 24668779]

24. Houston E, Argueta C, Shoptaw S. HIV and Depression: A Potential Role for Attention Training in Prevention and Treatment. J HIV AIDS 2018;4(2). [doi: 10.16966/2380-5536.152]

25. Fadardi J, Cox W, Klinger E. Individualized versus general measures of addiction-related implicit cognitions. In: Wiers RW, Stacy AW, editors. Handbook of Implicit Cognition and Addiction. Thousand Oaks, CA: Sage Publications; $2006: 121$.

26. Wisco BE. Depressive cognition: self-reference and depth of processing. Clin Psychol Rev 2009 Jun;29(4):382-392. [doi: 10.1016/j.cpr.2009.03.003] [Medline: 19346043]

27. Christopoulos K, Das M, Colfax G. Linkage and retention in HIV care among men who have sex with men in the United States. Clin Infect Dis. 52 Suppl 2, S 2011:214. [doi: 10.1093/cid/ciq045]

28. Giordano TP, Guzman D, Clark R, Charlebois ED, Bangsberg DR. Measuring Adherence to Antiretroviral Therapy in a Diverse Population Using a Visual Analogue Scale. HIV Clinical Trials 2015 Feb 25;5(2):74-79. [doi:

10.1310/jfxh-g3x2-eym6-d6ug]

29. Kalichman SC, Amaral CM, Swetzes C, Jones M, Macy R, Kalichman MO, et al. A simple single-item rating scale to measure medication adherence: further evidence for convergent validity. J Int Assoc Physicians AIDS Care (Chic) 2009 Dec 15;8(6):367-374 [FREE Full text] [doi: 10.1177/1545109709352884] [Medline: 19952289]

30. Gordon LL, Gharibian D, Chong K, Chun H. Comparison of HIV Virologic Failure Rates Between Patients with Variable Adherence to Three Antiretroviral Regimen Types. AIDS Patient Care STDS 2015 Jul;29(7):384-388. [doi: 10.1089/apc.2014.0165] [Medline: 26114665]

31. Kobin AB, Sheth NU. Levels of Adherence Required for Virologic Suppression Among Newer Antiretroviral Medications. Ann Pharmacother 2011 Mar 08;45(3):372-379. [doi: 10.1345/aph.1p587]

32. Shuter J. Forgiveness of non-adherence to HIV-1 antiretroviral therapy. J Antimicrob Chemother 2008 Apr 04;61(4):769-773. [doi: 10.1093/jac/dkn020] [Medline: 18256112]

33. Shuter J, Sarlo JA, Kanmaz TJ, Rode RA, Zingman BS. HIV-Infected Patients Receiving Lopinavir/Ritonavir-Based Antiretroviral Therapy Achieve High Rates of Virologic Suppression Despite Adherence Rates Less Than 95\%. JAIDS Journal of Acquired Immune Deficiency Syndromes 2007;45(1):4-8. [doi: 10.1097/qai.0b013e318050d8c2]

34. Viswanathan S, Justice AC, Alexander GC, Brown TT, Gandhi NR, McNicholl IR, et al. Adherence and HIV RNA Suppression in the Current Era of Highly Active Antiretroviral Therapy. JAIDS Journal of Acquired Immune Deficiency Syndromes 2015;69(4):493-498. [doi: 10.1097/qai.0000000000000643] 
35. Kroenke K, Spitzer RL, Williams JBW, Löwe B. The Patient Health Questionnaire Somatic, Anxiety, and Depressive Symptom Scales: a systematic review. Gen Hosp Psychiatry 2010 Jul;32(4):345-359. [doi: 10.1016/j.genhosppsych.2010.03.006] [Medline: 20633738]

36. Kroenke K, Spitzer RL, Williams JBW. The PHQ-9: validity of a brief depression severity measure. J Gen Intern Med 2001 Sep;16(9):606-613 [FREE Full text] [doi: 10.1046/j.1525-1497.2001.016009606.x] [Medline: 11556941]

37. Weathers F, Huska J, Keane T. PCL-C for DSM-IV. Boston, MA: National Center for PTSD, Behavioral Science Division 1991.

38. National CFP. Using the PTSD checklist for DSM IV (PCL). 2012. URL: https://www.ptsd.va.gov/professional/assessment/ documents/PCL handoutDSM4.pdf

39. Alexander MJ, Haugland G, Lin SP, Bertollo DN, McCorry FA. Mental Health Screening in Addiction, Corrections and Social Service Settings: Validating the MMS. Int J Ment Health Addiction 2007 Jun 14;6(1):105-119. [doi: $10.1007 / \mathrm{s} 11469-007-9100-\mathrm{x}]$

40. Jonzon E, Lindblad F. Risk factors and protective factors in relation to subjective health among adult female victims of child sexual abuse. Child Abuse Negl 2006 Feb;30(2):127-143. [doi: 10.1016/j.chiabu.2005.08.014] [Medline: 16466788]

41. Kalichman SC, Simbayi LC, Cloete A, Mthembu PP, Mkhonta RN, Ginindza T. Measuring AIDS stigmas in people living with HIV/AIDS: the Internalized AIDS-Related Stigma Scale. AIDS Care 2009 Jan 11;21(1):87-93. [doi: 10.1080/09540120802032627] [Medline: 19085224$]$

42. Cacioppo JT, von Hippel W, Ernst JM. Mapping cognitive structures and processes through verbal content: The thought-listing technique. Journal of Consulting and Clinical Psychology 1997;65(6):928-940. [doi: 10.1037/0022-006x.65.6.928]

43. Lease AM, McFall RM, Treat TA, Viken RJ. Assessing Children's Representations of their Peer Group Using a Multidimensional Scaling Technique. Journal of Social and Personal Relationships 2016 Jun 29;20(6):707-728. [doi: $10.1177 / 0265407503206001]$

44. Stacy AW, Newcomb MD, Ames SL. Implicit cognition and HIV risk behavior. J Behav Med 2000 Oct;23(5):475-499. [doi: 10.1023/a:1005577132666] [Medline: 11039159]

45. Jones L, Koehly L. Multidimensional scaling. Keren G, Lewis C. editors. A Handbook for Data Analysis in the Behavioral Sciences: methodological issues (pp.95-164). Hillsdale, NJ. Erlbaum 1993:E. [doi: 10.4324/9781315799582-9]

46. Compendium of evidence-based effective HIV behavioral interventions: Treatment Advocacy Program. Available at. URL: https://www.cdc.gov/hiv/pdf/research/interventionresearch/compendium/rr/TAP_BEST_RR.pdf

47. McKirnan DJ, Tolou-Shams M, Courtenay-Quirk C. The Treatment Advocacy Program: a randomized controlled trial of a peer-led safer sex intervention for HIV-infected men who have sex with men. J Consult Clin Psychol 2010 Dec;78(6):952-963 [FREE Full text] [doi: 10.1037/a0020759] [Medline: 20919760]

48. Raja S, McKirnan D, Glick N. The Treatment Advocacy Program--Sinai: a peer-based HIV prevention intervention for working with African American HIV-infected persons. AIDS Behav 2007 Sep 12;11(5 Suppl):S127-S137. [doi: 10.1007/s10461-007-9226-7] [Medline: 17436076]

49. Donaldson C, Lam D, Mathews A. Rumination and attention in major depression. Behav Res Ther 2007 Nov;45(11):2664-2678. [doi: 10.1016/j.brat.2007.07.002] [Medline: 17692819]

50. Cohen J. A power primer. Psychological Bulletin 1992;112(1):155-159. [doi: 10.1037/0033-2909.112.1.155]

51. Kaur J, Dodson JE, Steadman L, Vance DE. Predictors of improvement following speed of processing training in middle-aged and older adults with HIV: a pilot study. J Neurosci Nurs 2014 Feb;46(1):23-33 [FREE Full text] [doi: 10.1097/JNN.0000000000000034] [Medline: 24399164]

52. Vance DE, Fazeli PL, Ross LA, Wadley VG, Ball KK. Speed of processing training with middle-age and older adults with HIV: a pilot study. J Assoc Nurses AIDS Care 2012 Nov;23(6):500-510 [FREE Full text] [doi: 10.1016/j.jana.2012.01.005] [Medline: 22579081]

53. Amir N, Kuckertz JM, Strege MV. A Pilot Study of an Adaptive, Idiographic, and Multi-Component Attention Bias Modification Program for Social Anxiety Disorder. Cognit Ther Res 2016 Oct 7;40(5):661-671 [FREE Full text] [doi: 10.1007/s10608-016-9781-1] [Medline: 27795598]

54. Grafton B, Mackintosh B, Vujic T, MacLeod C. When Ignorance is Bliss: Explicit Instruction and the Efficacy of CBM-A for Anxiety. Cogn Ther Res 2013 Sep 11;38(2):172-188. [doi: 10.1007/s10608-013-9579-3]

55. Krebs G, Hirsch CR, Mathews A. The effect of attention modification with explicit vs. minimal instructions on worry. Behav Res Ther 2010 Mar;48(3):251-256. [doi: 10.1016/j.brat.2009.10.009] [Medline: 19926075]

56. Kuckertz JM, Amir N. Attention bias modification for anxiety and phobias: current status and future directions. Curr Psychiatry Rep 2015 Feb 27;17(2):9. [doi: 10.1007/s11920-014-0545-x] [Medline: 25620791]

57. Lazarov A, Abend R, Seidner S, Pine DS, Bar-Haim Y. The Effects of Training Contingency Awareness During Attention Bias Modification on Learning and Stress Reactivity. Behav Ther 2017 Sep;48(5):638-650 [FREE Full text] [doi: 10.1016/j.beth.2017.03.002] [Medline: 28711114]

58. MacLeod C, Clarke PJF. The Attentional Bias Modification Approach to Anxiety Intervention. Clinical Psychological Science 2015 Jan 06;3(1):58-78. [doi: 10.1177/2167702614560749] 
59. Mobini S, Mackintosh B, Illingworth J, Gega L, Langdon P, Hoppitt L. Effects of standard and explicit cognitive bias modification and computer-administered cognitive-behaviour therapy on cognitive biases and social anxiety. J Behav Ther Exp Psychiatry 2014 Jun;45(2):272-279 [FREE Full text] [doi: 10.1016/j.jbtep.2013.12.002] [Medline: 24412966]

60. Nishiguchi Y, Takano K, Tanno Y. Explicitly guided attentional bias modification promotes attentional disengagement from negative stimuli. Emotion 2015;15(6):731-741. [doi: 10.1037/a0039117]

61. Phillips WJ, Hine DW, Thorsteinsson EB. Implicit cognition and depression: a meta-analysis. Clin Psychol Rev 2010 Aug;30(6):691-709. [doi: 10.1016/j.cpr.2010.05.002] [Medline: 20538393]

62. Vazquez C, Blanco I, Sanchez A, McNally RJ. Attentional bias modification in depression through gaze contingencies and regulatory control using a new eye-tracking intervention paradigm: study protocol for a placebo-controlled trial. BMC Psychiatry 2016 Dec 08;16(1):439 [FREE Full text] [doi: 10.1186/s12888-016-1150-9] [Medline: 27931196]

63. Locke E, Latham G. Englewood Cliffs, NJ. US: Prentice-Hall, Inc 1990.

64. Simoni JM, Kurth AE, Pearson CR, Pantalone DW, Merrill JO, Frick PA. Self-report measures of antiretroviral therapy adherence: A review with recommendations for HIV research and clinical management. AIDS Behav 2006 May 3;10(3):227-245 [FREE Full text] [doi: 10.1007/s10461-006-9078-6] [Medline: 16783535]

65. Friese M, Hofmann W, Wiers RW. On taming horses and strengthening riders: Recent developments in research on interventions to improve self-control in health behaviors. Self and Identity 2011 May 19;10(3):336-351. [doi: $\underline{10.1080 / 15298868.2010 .536417]}$

\section{Abbreviations \\ PHQ: Patient Health Questionnaire \\ PTSD: posttraumatic stress disorder}

Edited by A Tsanas, G Eysenbach; submitted 19.02.20; peer-reviewed by I Wani, B Smith; comments to author $17.03 .20 ;$ revised
version received 31.03.20; accepted 03.04.20; published 28.01.21
Please cite as:
Houston E, Fadardi JS, Harawa NT, Argueta C, Mukherjee $S$
Individualized Web-Based Attention Training With Evidence-Based Counseling to Address HIV Treatment Adherence and Psychological
Distress: Exploratory Cohort Study
JMIR Ment Health 2021;8(1):e18328
URL: $\underline{\text { http://mental.jmir.org/2021/1/e18328/ }}$
doi: $\underline{10.2196 / 18328}$
PMID: $\underline{33507152}$

CEric Houston, Javad Salehi Fadardi, Nina T Harawa, Chris Argueta, Sukrit Mukherjee. Originally published in JMIR Mental Health (http://mental.jmir.org), 28.01.2021. This is an open-access article distributed under the terms of the Creative Commons Attribution License (https://creativecommons.org/licenses/by/4.0/), which permits unrestricted use, distribution, and reproduction in any medium, provided the original work, first published in JMIR Mental Health, is properly cited. The complete bibliographic information, a link to the original publication on http://mental.jmir.org/, as well as this copyright and license information must be included. 